\title{
INVARIANT MEASURES ON NILPOTENT ORBITS ASSOCIATED WITH HOLOMORPHIC DISCRETE SERIES
}

\author{
MLADEN BOŽIČEVIĆ
}

\begin{abstract}
Let $G_{\mathbb{R}}$ be a real form of a complex, semisimple Lie group $G$. Assume $G_{\mathbb{R}}$ has holomorphic discrete series. Let $\mathcal{W}$ be a nilpotent coadjoint $G_{\mathbb{R}}$-orbit contained in the wave front set of a holomorphic discrete series. We prove a limit formula, expressing the canonical measure on $\mathcal{W}$ as a limit of canonical measures on semisimple coadjoint orbits, where the parameter of orbits varies over the positive chamber defined by the Borel subalgebra associated with holomorphic discrete series.
\end{abstract}

\section{INTRODUCTION}

Let $G_{\mathbb{R}}$ be a semisimple Lie group, $\mathfrak{g}_{\mathbb{R}}$ the Lie algebra of $G_{\mathbb{R}}, \mathfrak{g}$ the complexification of $\mathfrak{g}_{\mathbb{R}}$, and $X$ the flag variety of $\mathfrak{g}$.

It was first observed by Rossmann in the setting of complex groups, and later refined and generalized by Schmid and Vilonen SV2] to arbitrary semisimple groups, that invariant eigendistributions on the Lie algebra can be expressed as integrals of certain equivariant forms over the homology cycles supported on the conormal variety of $G_{\mathbb{R}^{-}}$action on $X$. Such integral formulas have proved to be useful in studying asymptotic properties of invariant eigendistributions, and, in particular, for computing the Liouville measure on a coadjoint nilpotent orbit in terms of Liouville measures on regular semisimple orbits. The corresponding formulas are known as limit formulas. They already appear in the classical work of Harish-Chandra on the harmonic analysis on semisimple groups. Namely, the simplest example of limit formulas is the Harish-Chandra's formula for delta function at zero.

The main goal of the present paper is to apply the results of Schmid and Vilonen SV2, [SV3] and of Rossmann [Ro1, Ro2, Ro3, to compute Liouville measures on the nilpotent orbits contained in the wave front set of holomorphic discrete series. In fact, one can deduce from the theory of Schmid and Vilonen that the wave front set of discrete series representations is the closure of a single nilpotent $G_{\mathbb{R}^{-o r b i t}}$ [Bo1]. In the case of holomorphic discrete series the orbit structure of the wave front set is relatively simple: distinct real nilpotent orbits contained in the wave front set are not conjugated under the complexification of the real group. Furthermore the homology group of $G_{\mathbb{R}}$-equivariant cycles supported on the inverse image of the wave front set under the moment map is generated as a $W$-module by the characteristic cycle of the standard sheaf associated with discrete series representations. These properties of the wave front set of the holomorphic discrete series can be used to

Received by the editors January 26, 2021, and, in revised form, May 25, 2021.

2020 Mathematics Subject Classification. Primary 22E46; Secondary 22E30.

The author was partially supported by grant no. 4176 of the Croatian Science Foundation. 
obtain limit formulas by the method very similar to the one applied in [Bo3] in the case of real groups with one conjugacy class of Cartan subgroups.

A considerable amount of results is needed to prove Theorem 4.6, the main result of the paper. We have made an effort to review these results throughout the paper. In the first two sections we recall the construction of the invariant distributions associated with invariant cycles in the conormal bundle of the flag variety, and Rossmann's theory of Weyl group representations on the homology groups of conormal varieties. In the fourth section we review the theory of Schmid and Vilonen on the microlocalization of the Matsuki correspondence between $K$ equivariant and $G_{\mathbb{R}^{-}}$equivariant sheaves. The paper probably doesn't open new possibilities for proving limit formulas for general nilpotent orbit, but hopefully it serves as a new and relevant example of application of the powerful theory developed by Schmid and Vilonen to the problems of harmonic analysis on semisimple Lie groups.

\section{Preliminaries}

Suppose $G_{\mathbb{R}}$ is a real, connected, linear, semisimple Lie group. We embed $G_{\mathbb{R}}$ into a complexification $G$ and denote by

$$
\tau: G \longrightarrow G
$$

the involution on $G$ having $G_{\mathbb{R}}$ as the identity component of the set of fixed points. Next we choose a Cartan involution

$$
\theta: G_{\mathbb{R}} \longrightarrow G_{\mathbb{R}}
$$

and extend it to $G$. Denote by $K_{\mathbb{R}}$ resp. $K$ the set of fixed points of $\theta$ on $G_{\mathbb{R}}$ resp. $G$. Observe that $\theta \tau$ is a Cartan involution on $G$. We denote by $U_{\mathbb{R}}$ the set of fixed points. Write $\mathfrak{g}, \mathfrak{k}, \mathfrak{g}_{\mathbb{R}}, \mathfrak{k}_{\mathbb{R}}, \mathfrak{u}_{\mathbb{R}}$ for the Lie algebras of $G, K, G_{\mathbb{R}}, K_{\mathbb{R}}, U_{\mathbb{R}}$ respectively. Denote the involutions on $\mathfrak{g}$ induced by $\theta, \tau$ by the same letters. In addition, let

$$
\mathfrak{g}_{\mathbb{R}}=\mathfrak{k}_{\mathbb{R}}+\mathfrak{p}_{\mathbb{R}}, \mathfrak{g}=\mathfrak{k}+\mathfrak{p}
$$

be the eigenspace decompositions defined by $\theta$. Let $($,$) be the Killing form on \mathfrak{g}$. We will use it whenever convenient to identify $\mathfrak{g}$ and the dual space $\mathfrak{g}^{*}$.

Next we introduce the notation related to the geometry of the flag variety. Write $X$ for the flag variety of Borel subalgebras of $\mathfrak{g}$. Let

$$
n=\operatorname{dim}_{\mathbb{C}} X .
$$

Given $x \in X$ we denote by $\mathfrak{b}_{x}$ the corresponding Borel subalgebra, and by $B_{x} \subset$ $G$ the Borel subgroup which stabilizes $x$ via the adjoint action. Consider $G$ homogenous bundles $\mathcal{B}$ and $[\mathcal{B}, \mathcal{B}]$ over $X$ with fiber $\mathfrak{b}_{x}$ resp. $\left[\mathfrak{b}_{x}, \mathfrak{b}_{x}\right]$ at $x \in X$. Observe that $B_{x}$ acts trivially on $\mathfrak{b}_{x} /\left[\mathfrak{b}_{x}, \mathfrak{b}_{x}\right]$; hence the $G$-bundle $\mathcal{B} /[\mathcal{B}, \mathcal{B}]$ is trivial. We denote by $\mathfrak{h}$ its fiber, and call it the universal Cartan subalgebra. Note that $\mathfrak{h} \simeq \mathfrak{b}_{x} /\left[\mathfrak{b}_{x}, \mathfrak{b}_{x}\right]$ canonically, for any $x \in X$. Let $\mathfrak{c} \subset \mathfrak{g}$ be a Cartan subalgebra. Denote by $\Delta(\mathfrak{g}, \mathfrak{c})$ the root system. Then $\mathfrak{c}$ has $|W|$ fixed points on $X(||$ stands for the cardinality), and we choose one of them: $x \in X$. Then $\mathfrak{c} \subset \mathfrak{b}_{x}$, and we have a canonical isomorphism $\tau_{x}: \mathfrak{c} \rightarrow \mathfrak{h}$. We denote by $\tau_{x}^{*}: \mathfrak{h}^{*} \rightarrow \mathfrak{c}^{*}$ the dual isomorphism. Then $\Delta=\tau_{x}^{*-1}(\Delta(\mathfrak{g}, \mathfrak{c}))$ is independent of the choice of the pair $(\mathfrak{c}, x)$, and is called the universal root system. Set $\Delta_{x}^{+}=\Delta\left(\mathfrak{g} / \mathfrak{b}_{x}, \mathfrak{c}\right)$. A positive root system in $\Delta$ is defined by the condition

$$
\Delta^{+}=\tau_{x}^{*-1}\left(\Delta_{x}^{+}\right)
$$


Given $\lambda \in \Delta$, and a pair $(\mathfrak{c}, x)$ as above, we write $\lambda_{x}=\tau_{x}^{*}(\lambda)$. The universal Weyl group $W$ is defined as the Weyl group of the root system $\Delta$. Denote by $\rho \in \mathfrak{h}^{*}$ half the sum of the positive roots, and by $\mathfrak{h}^{* \prime}$ the set of regular elements. Note that $\mathfrak{h}$ resp. $\mathfrak{h}^{*}$ comes equipped with $W$-invariant symmetric bilinear form $(\cdot, \cdot)$ whose specialization at $x \in X$ coincides with the Killing form. In particular, if $\lambda \in \mathfrak{h}^{*}$ we write $h_{\lambda}$ for the element in $\mathfrak{h}$ such that $\lambda(h)=\left(h, h_{\lambda}\right), h \in \mathfrak{h}$.

Let us recall the definition of the moment map and of the twisted moment map. Denote by $T^{*} X$ the cotangent bundle of the variety $X$. Given $x \in X$, denote by $\mathfrak{b}_{x}^{\perp} \subset \mathfrak{g}^{*}$ the space of linear forms vanishing on $\mathfrak{b}_{x}$. We use the identification

$$
T^{*} X \cong\left\{(x, \xi): x \in X, \xi \in \mathfrak{b}_{x}^{\perp}\right\},
$$

to consider $T^{*} X$ as a submanifold of $X \times \mathfrak{g}^{*}$ The moment map is defined by

$$
\mu: T^{*} X \longrightarrow \mathfrak{g}^{*}, \quad \mu(x, \xi)=\xi .
$$

Denote by $\mathcal{N}^{*}$ the cone of nilpotent elements in $\mathfrak{g}^{*}$. Note that $\mu\left(T^{*} X\right)=\mathcal{N}^{*}$. The definition of the twisted moment map is due to Rossmann Ro1. Note that any $x \in X$ is fixed by a unique maximal torus $C_{\mathbb{R}} \subset U_{\mathbb{R}}$. We can use the decomposition $\mathfrak{g}=\mathfrak{c}+[\mathfrak{c}, \mathfrak{g}]$ to view $\mathfrak{c}^{*}$ as a subspace of $\mathfrak{g}^{*}$. Now we define the twisted moment map by

$$
\mu_{\lambda}: T^{*} X \longrightarrow \mathfrak{g}^{*}, \mu_{\lambda}(x, \xi)=\lambda_{x}+\mu(x, \xi), \quad \xi \in \mathfrak{b}_{x}^{\perp} .
$$

If $\lambda$ is regular, one can show that $\mu_{\lambda}$ is a $U_{\mathbb{R}}$-equivariant, real algebraic isomorphism of $T^{*} X$ with complex coadjoint orbit $A d^{*}(G) \lambda_{x}$. Note that $A d^{*}(G) \lambda_{x}$ is independent of $x \in X$. We shall write $G \cdot \lambda=A d^{*}(G) \lambda_{x}$.

Let $\mathcal{V}$ be a coadjoint $G$-orbit in $\mathfrak{g}^{*}$ or a coadjoint $G_{\mathbb{R}}$-orbit in $i \mathfrak{g}_{\mathbb{R}}^{*}$. To treat both cases simultaneously write $M=G$ or $M=G_{\mathbb{R}}$ and denote by $\mathfrak{m}$ the Lie algebra of $M$. The space

$$
\mathfrak{m} \cdot \xi=\left\{\operatorname{ad}^{*}(x)(\xi): x \in \mathfrak{m}\right\}
$$

identifies with tangent space $T_{\xi} \mathcal{V}$ of $\mathcal{V}$ at $\xi$, and we define a $M$-equivariant 2-form $\sigma_{\mathcal{V}}$ on $\mathcal{V}$ by the formula

$$
\sigma_{\mathcal{V}, \xi}(x \cdot \xi, y \cdot \xi)=\xi[x, y], \quad x, y \in \mathfrak{m} .
$$

In case $M=G_{\mathbb{R}}$ the form $-i \sigma_{\mathcal{V}}$ is real valued and we use the form

$$
\left(-i \sigma_{\mathcal{V}}\right)^{k}, 2 k=\operatorname{dim}_{\mathbb{R}} \mathcal{V}
$$

to orient $\mathcal{V}$. In this case we define the measure $m_{\mathcal{V}}$ by the formula

$$
d m_{\mathcal{V}}=\frac{1}{(2 \pi i)^{k} k !} \sigma_{\mathcal{V}}^{k}
$$

and call it the Liouville measure. When $\mathcal{V}=M \cdot \lambda, \lambda \in \mathfrak{c}^{*}, \mathfrak{c} \subset \mathfrak{g}$ a Cartan subalgebra, we shall write $\sigma_{\mathcal{V}}=\sigma_{\lambda}$.

Let $\lambda \in \mathfrak{h}^{*}$. Then a $U_{\mathbb{R}^{-}}$equivariant 2 -form $\tau_{\lambda}$ on $X$ is defined at $x$ by

$$
\tau_{\lambda}\left(a_{x}, b_{x}\right)=\tau_{x}^{*}(\lambda)([a, b]) .
$$

Here $a_{x}$ and $b_{x}$ denote the tangent vectors induced by $a, b \in \mathfrak{u}_{\mathbb{R}}$ by differentiation

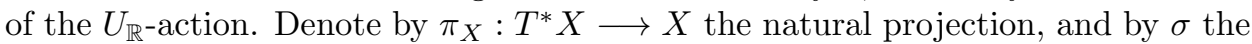
canonical symplectic form on $T^{*} X$. For $\lambda \in \mathfrak{h}^{* \prime}$ the following formula holds [SV2]:

$$
\mu_{\lambda}^{*}\left(\sigma_{\lambda}\right)=-\sigma+\pi_{X}^{*}\left(\tau_{\lambda}\right) .
$$


Next we recall, following [SV2, the definition of invariant distributions on the Lie algebra as integrals of certain differential forms over the semi-algebraic cycles in $T^{*} X$. The Fourier transform of a test function $\phi \in C_{c}^{\infty}\left(\mathfrak{g}_{\mathbb{R}}\right)$ will be defined by

$$
\hat{\phi}(\xi)=\int_{\mathfrak{g}_{\mathbb{R}}} e^{\xi(x)} \phi(x) d x, \xi \in \mathfrak{g}^{*},
$$

without the usual $i$ in the exponential. Here $d x$ denotes a suitably normalized Lebesgue measure on $\mathfrak{g}_{\mathbb{R}}$. Let $\Gamma$ be a semi-algebraic chain in $T^{*} X$. We say that $\Gamma$ is $\mathbb{R}$-bounded if

$$
\operatorname{Re} \mu(\operatorname{supp}(\Gamma)) \subset \mathfrak{g}^{*}
$$

is bounded. Here Re is defined with respect to $\mathfrak{g}_{\mathbb{R}}^{*}$. If $\Gamma$ is a semi-algebraic, $\mathbb{R}$ bounded, $2 n$-chain in $T^{*} X$ one can prove that for a test function $\phi \in C_{c}^{\infty}\left(\mathfrak{g}_{\mathbb{R}}\right)$ and $\lambda \in \mathfrak{h}^{*}$ the integral

$$
\Theta(\Gamma, \lambda)(\phi)=\int_{\Gamma} \mu_{\lambda}^{*}(\hat{\phi})\left(-\sigma+\pi_{X}^{*} \tau_{\lambda}\right)^{n}
$$

converges and depends holomorphically on $\lambda$. In particular, this is true for a cycle $\Gamma \in H_{2 n}\left(T_{G_{\mathbb{R}}}^{*} X, \mathbb{C}\right)$, where $T_{G_{\mathbb{R}}}^{*} X$ denotes the union of conormal bundles to the $G_{\mathbb{R}^{-}}$-orbits on $X$. In this case $\Theta(\Gamma, \lambda)$ is a $G_{\mathbb{R}^{-}}$invariant distribution on $\mathfrak{g}_{\mathbb{R}}$.

\section{WEYL GROUP REPRESENTATIONS}

In this section we recall the results of Rossmann on Weyl group representations on homology groups of conormal varieties for the group actions on the flag variety. Let

$$
A=K \text { or } A=G_{\mathbb{R}},
$$

and let $\mathfrak{a}$ be the Lie algebra of $A$. When

$$
S \subset \mathfrak{g}^{*}
$$

satisfies certain natural assumptions Ro2, Rossmann defines $W$-module structure on homology groups

$$
H_{*}\left(\mu^{-1}(S), \mathbb{C}\right) .
$$

In particular, we obtain $W$-modules in the following cases:

$$
S=\mathfrak{a}^{\perp} \cap \mathcal{N}^{*}, S=\overline{\mathcal{O}}, S=\mathcal{O}, S=\{\nu\} .
$$

Here $\mathcal{O}$ is an $A$-orbit and $\nu \in \mathcal{N}^{*}$. In the first case we have

$$
\mu^{-1}\left(\mathfrak{a}^{\perp} \cap \mathcal{N}^{*}\right)=T_{A}^{*} X .
$$

Rossmann shows Ro2 that inclusions of the $A$-orbit closures are compatible with $W$-module structure on homology groups. In fact,

(2) $\quad 0 \longrightarrow H_{2 n}\left(\mu^{-1}(\overline{\mathcal{O}} \backslash \mathcal{O}), \mathbb{C}\right) \longrightarrow H_{2 n}\left(\mu^{-1}(\overline{\mathcal{O}}), \mathbb{C}\right) \longrightarrow H_{2 n}\left(\mu^{-1}(\mathcal{O}), \mathbb{C}\right) \longrightarrow 0$

is an exact sequence of $W$-modules. Denote by

$$
C_{G}(\nu) \text { resp. } C_{A}(\nu)
$$

the group of connected components of the centralizer of $\nu$ in $G$ resp. A. Let

$$
d=d(\nu)=\operatorname{dim}_{\mathbb{C}} \mu^{-1}(\nu) .
$$

Then $C_{G}(\nu)$ acts on $H_{2 d}\left(\mu^{-1}(\nu), \mathbb{C}\right)$ by permuting the irreducible components, and this action commutes with $W$-action. Hence

$H_{2 d}\left(\mu^{-1}(\nu), \mathbb{C}\right)^{C_{G}(\nu)} \subset H_{2 d}\left(\mu^{-1}(\nu), \mathbb{C}\right)$ and $H_{2 d}\left(\mu^{-1}(\nu), \mathbb{C}\right)^{C_{A}(\nu)} \subset H_{2 d}\left(\mu^{-1}(\nu), \mathbb{C}\right)$ 
are $W$-submodules, and the natural projection

$$
H_{2 d}\left(\mu^{-1}(\nu), \mathbb{C}\right)^{C_{A}(\nu)} \longrightarrow H_{2 d}\left(\mu^{-1}(\nu), \mathbb{C}\right)^{C_{G}(\nu)}
$$

is a homomorphism of $W$-modules. Recall that the $W$-module $H_{2 d}\left(\mu^{-1}(\nu), \mathbb{C}\right)^{C_{G}(\nu)}$ is irreducible Ro1]. This is the Springer representation associated to the orbit $G \cdot \nu$, and we denote the corresponding character by $\chi_{\nu}$. We will also need the following isomorphism of $W$-modules Ro1]:

$$
H_{2 n}\left(\mu^{-1}(\mathcal{O}), \mathbb{C}\right) \cong H_{2 d}\left(\mu^{-1}(\nu), \mathbb{C}\right)^{C_{A}(\nu)} .
$$

Moreover, Rossmann's definition of $W$-action on $H_{2 n}\left(T_{G_{\mathbb{R}}}^{*} X, \mathbb{C}\right)$ implies the following $W$-equivariance formula for distributions $\Theta(\Gamma, \lambda)$ Ro1]:

$$
\Theta(w \Gamma, \lambda)=\Theta\left(\Gamma, w^{-1} \lambda\right), w \in W, \lambda \in \mathfrak{h}^{*} .
$$

Our goal is to study the asymptotic behaviour of distributions $\Theta(\Gamma, \lambda)$ when $\lambda \in \mathfrak{h}^{*}$ approaches zero. We recall additional facts needed for this analysis.

Denote by $\mathcal{H}_{d}\left(\mathfrak{h}^{*}\right)\left(\mathcal{H}_{d}(\mathfrak{h})\right)$ the space of harmonic polynomials on $\mathfrak{h}^{*}(\mathfrak{h})$ of degree d. The map

$$
H_{2 d}(X, \mathbb{C}) \longrightarrow \mathcal{H}_{d}\left(\mathfrak{h}^{*}\right), \gamma \mapsto b(\gamma)=\frac{1}{(2 \pi i)^{d} d !} \int_{\gamma} \tau_{\lambda}^{d}
$$

is an isomorphism of $W$-modules, usually called the Borel isomorphism Bor1. Here we consider the $W$-action on $H_{2 d}(X, \mathbb{C})$ induced by the natural $W$-action on $X$. On the other hand, we have a natural homomorphism

$$
H_{2 d}\left(\mu^{-1}(\nu), \mathbb{C}\right) \longrightarrow H_{2 d}(X, \mathbb{C}),
$$

defined by the inclusion $\mu^{-1}(\nu) \longrightarrow X \times\{\nu\}$. Rossmann shows this is a nonzero $W$-module homomorphism [Ro1] which factors through the projection

$$
H_{2 d}\left(\mu^{-1}(\nu), \mathbb{C}\right) \longrightarrow H_{2 d}\left(\mu^{-1}(\nu), \mathbb{C}\right)^{C_{G}(\nu)} \text {. }
$$

It is known that $\chi_{\nu}$ appears exactly once in $\mathcal{H}_{d}\left(\mathfrak{h}^{*}\right)$ [BM]. We denote the corresponding subspace by $\mathcal{H}_{d}\left(\mathfrak{h}^{*}\right)_{\nu}$. Now taking into the account (2), (3), (5), (6) and (7) we obtain a surjective homomorphism of $W$-modules

$$
H_{2 n}\left(\mu^{-1}(\overline{\mathcal{O}}), \mathbb{C}\right) \longrightarrow \mathcal{H}_{d}\left(\mathfrak{h}^{*}\right)_{\nu}, \Gamma \mapsto p_{\Gamma}
$$

Denote by $\Theta_{\mathcal{O}}$ the Fourier transform of the Liouville measure $m_{\mathcal{O}}$. In more details,

$$
\Theta_{\mathcal{O}}(\phi)=\frac{1}{(2 \pi i)^{k} k !} \int_{\mathcal{O}} \hat{\phi} \sigma_{\mathcal{O}}^{k}, \quad 2 k=\operatorname{dim}_{\mathbb{R}} \mathcal{O}, \phi \in C_{c}^{\infty}\left(\mathfrak{g}_{\mathbb{R}}\right) .
$$

Let $\Gamma \in H_{2 n}\left(\mu^{-1}(\overline{\mathcal{O}}), \mathbb{C}\right)$. Rossmann proves Ro1 the following formula relating distributions $\Theta(\Gamma, \lambda)$ and $\Theta_{\mathcal{O}}$

$$
\Theta(\Gamma, \lambda)=p_{\Gamma}(\lambda) \Theta_{\mathcal{O}}+o\left(\lambda^{n-k}\right) .
$$

The term $o\left(\lambda^{n-k}\right)$ can be described as follows. For any $\phi \in C_{c}^{\infty}\left(\mathfrak{g}_{\mathbb{R}}\right), o\left(\lambda^{n-k}\right)(\phi)$ is a holomorphic function of $\lambda$ and

$$
\lim _{t \rightarrow 0} \frac{o\left((t \lambda)^{n-k}\right)(\phi)}{t^{n-k}}=0 .
$$

Finally we recall two results from Bo3 that will be needed in the proof of the main result. Denote by $\mathbb{C}[\mathfrak{h}]$ resp. $\mathbb{C}\left[\mathfrak{h}^{*}\right]$ the algebra of polynomial functions on $\mathfrak{h}$ 
resp. $\mathfrak{h}^{*}$. Write $S(\mathfrak{h})$ resp. $S\left(\mathfrak{h}^{*}\right)$ for the symmetric algebra of $\mathfrak{h}$ resp. $\mathfrak{h}^{*}$. Recall that we have canonical isomorphisms

$$
\mathbb{C}[\mathfrak{h}] \cong S\left(\mathfrak{h}^{*}\right) \text { and } \mathbb{C}\left[\mathfrak{h}^{*}\right] \cong S(\mathfrak{h}) .
$$

On the other hand the map

$$
v \mapsto \partial(v), \quad \partial(v) f(\lambda)=\lim _{t \rightarrow 0}(f(\lambda+t v)-f(\lambda)) / t, \quad \lambda, v \in \mathfrak{h}^{*}, f \in C^{\infty}\left(\mathfrak{h}^{*}\right)
$$

extends to an isomorphism of $S\left(\mathfrak{h}^{*}\right)$ and the algebra $D\left(\mathfrak{h}^{*}\right)$ of differential operators on $\mathfrak{h}^{*}$ with constant coefficients. Thus we obtain the isomorphism of algebras

$$
\mathbb{C}[\mathfrak{h}] \cong D\left(\mathfrak{h}^{*}\right), \quad p \mapsto p(\partial), p \in \mathbb{C}[\mathfrak{h}] .
$$

Let $\delta: \mathfrak{h} \longrightarrow \mathfrak{h}^{*}$ be the isomorphism defined by the Killing form and

$$
\delta: S(\mathfrak{h}) \longrightarrow S\left(\mathfrak{h}^{*}\right)
$$

the induced isomorphism of algebras. As usual we write

$$
\delta^{-1}(\lambda)=h_{\lambda}, \lambda \in \mathfrak{h}^{*} .
$$

Put

$$
\mathfrak{h}_{0}^{*}=\sum_{\alpha \in \Delta^{+}} \mathbb{R} \cdot \alpha
$$

and denote by

$$
{ }^{-}: \mathfrak{h}^{*} \longrightarrow \mathfrak{h}^{*}
$$

the conjugation with respect to $\mathfrak{h}_{0}^{*}$. Let

$$
{ }^{-}: S\left(\mathfrak{h}^{*}\right) \longrightarrow S\left(\mathfrak{h}^{*}\right)
$$

be the induced conjugation of $S\left(\mathfrak{h}^{*}\right)$.

Lemma 2.1. Let $\left(r_{1}, \cdots, r_{s}\right)$ be a basis in $\mathcal{H}_{d}\left(\mathfrak{h}^{*}\right)_{\nu} \subset S(\mathfrak{h})$. Put $p_{i}=\delta\left(r_{i}\right)$, $i=1, \cdots, s$ and let

$$
V_{d}=\sum_{i=1}^{s} \mathbb{C} \cdot \bar{p}_{i} .
$$

Then $V_{d}$ is a $W$-module isomorphic to $\mathcal{H}_{d}\left(\mathfrak{h}^{*}\right)_{\nu}$.

Lemma 2.2. Let $\Gamma \in H_{2 n}\left(T_{\mathbb{R}}^{*} X, \mathbb{C}\right), \lambda \in \mathfrak{h}^{*}, p \in \mathbb{C}[\mathfrak{h}]$ and $w \in W$.

(1) $\lim _{\lambda \rightarrow 0} p(\partial) \Theta(\Gamma, \lambda)$ exists as a distribution on $\mathfrak{g}_{\mathbb{R}}$.

(2) $\lim _{\lambda \rightarrow 0} w^{-1} p(\partial) \Theta(\Gamma, \lambda)=\lim _{\lambda \rightarrow 0} p(\partial) \Theta(w \Gamma, \lambda)$.

\section{P-equivariant sheaves on the flag Variety}

Denote by $\Pi$ the set of simple roots in $\Delta^{+}$. Let $\Theta \subset-\Pi$. Denote by $W_{\Theta}$ the subgroup of $W$ generated by reflections $s_{\alpha}, \alpha \in \Theta$. The set

$$
W^{\Theta}=\left\{w \in W: \Theta \subset w\left(-\Delta^{+}\right)\right\}
$$

is a section of right $W_{\Theta}$-cosets consisting of elements of shortest length. Choose a point $x_{0} \in X$. Let $\mathfrak{c} \subset \mathfrak{b}_{x_{0}}$ be a Cartan subalgebra. Consider the isomorphism

$$
\mathfrak{h} \longrightarrow \mathfrak{b}_{x_{0}} /\left[\mathfrak{b}_{x_{0}}, \mathfrak{b}_{x_{0}}\right] \cong \mathfrak{c}
$$

It induces the isomorphism $W \longrightarrow W_{x_{0}}, w \mapsto w_{x_{0}}$, of the abstract Weyl group $W$ and the Weyl group $W_{x_{0}}$ of the pair $(\mathfrak{g}, \mathfrak{c})$. Denote by $B$ the Borel subgroup of $G$ with Lie algebra $\mathfrak{b}_{x_{0}}$, and by $P$ the standard parabolic subgroup of $G$ of type $\Theta_{x_{0}}$ containing $B$. Here $\Theta_{x_{0}}$ denotes the inverse image of $\Theta$ under the isomorphism 
$\mathfrak{c}^{*} \rightarrow \mathfrak{h}^{*}$. Let $\mathfrak{b}_{x_{w}}$ be the Borel subalgebra defined by the pair $\left(\mathfrak{c}, w_{x_{0}} \Delta\left(\mathfrak{c}, \mathfrak{b}_{x_{0}}\right)\right)$. Let $D(w)=P . x_{w}$. It is well known Mi] that the map

$$
W^{\Theta} \longrightarrow P \backslash X
$$

is a bijection between $W^{\Theta}$ and the set of all $P$-orbits on $X$.

Given a semi-algebraic set $Y$ we denote by $D(Y)$ the bounded derived category of sheaves of (complex) vector spaces constructible for semi-algebraic stratifications on $Y$. Let $f: Y \longrightarrow Z$ be a semi-algebraic map of (locally compact) semi-algebraic sets. Then the notation for functors

$$
\begin{gathered}
R f_{*}: D(Y) \longrightarrow D(Z), R f_{!}: D(Y) \longrightarrow D(Z), \\
f^{-1}: D(Z) \longrightarrow D(Y), f^{!}: D(Z) \longrightarrow D(Y)
\end{gathered}
$$

is the same as in $[\mathrm{KS}$. Suppose that $A$ is a real algebraic group acting on $Y$. The construction of Bernstein and Lunts $\left[\mathrm{BL}\right.$ associates to $D_{c}^{b}(Y)$ the category $D_{A}(Y)$ of $A$-equivariant sheaves on $X$. If $f: Y \longrightarrow Z$ is an $A$-equivariant map then the functors $f^{-1}, f^{!}, R f_{*}$ and $R f_{\text {! }}$ lift to functors between $A$-equivariant derived categories.

We return now to the setting of flag variety. Let $A \subset G$ be a real algebraic subgroup acting with finitely many orbits on the flag variety $X$. It is known [SV2, 6.3 , that the $K$-group $K\left(D_{A}(X)\right)$ is generated by standard sheaves. We recall the definition. Let $S \subset X$ be an $A$-orbit and $\tau$ an irreducible $A$-equivariant local system on $S$. To the pair $(S, \tau)$ we associate the standard sheaf

$$
\mathcal{I}(S, \tau)=R i_{S !}(\tau) \text {. }
$$

Here $i_{S}: S \longrightarrow X$ denotes the inclusion map. We describe in more details $A$ equivariant local systems on the orbit $S$. Recall that irreducible $A$-equivariant local systems on $S$ are parametrized by irreducible representations of $Z_{A(x)} / Z_{A}(x)^{\circ}$, the group of connected components of the centralizer of $x \in S$ in $A$. In particular, if $Z_{A(x)}$ is connected $\mathbb{C}_{S}$ is the unique irreducible $A$-equivariant local system on $S$.

We assume now that the number of $A$-orbits on $Y$ is finite, as will be the case in our applications. Next we relate the $K$-group of the $A$-equivariant sheaves to the characteristic cycle construction. In order to explain this, we need some additional notation. Recall that $H_{i}(Y, \mathbb{Z})$ resp. $H_{i}(Y, \mathbb{C}), i \in \mathbb{Z}$ denote the Borel-Moore homology groups with integral resp. complex coefficients. Suppose that $Y$ is a real algebraic manifold. The characteristic cycle $C C(\mathcal{F})$ of a constructible sheaf $\mathcal{F}$ from $D(Y)$ was defined by Kashiwara [KS] as a Lagrangian cycle in the real cotangent bundle $T^{*} Y$. In fact, let $\mathcal{S}$ be a semi-algebraic Whitney stratification on $\mathrm{Y}$, and $\mathcal{F}$ a complex of sheaves on $Y$ constructible for $\mathcal{S}$. Denote by $T_{\mathcal{S}}^{*} Y$ the union of conormal bundles to the strata. Then

$$
C C(\mathcal{F}) \in H_{m}\left(T_{\mathcal{S}}^{*} Y, \mathbb{Z}\right), \quad m=\operatorname{dim}_{\mathbb{R}} Y .
$$

In particular, if $\mathcal{F}$ is constructible for the orbit stratification, and if we denote by $T_{A}^{*} Y$ the union of conormal bundles to the orbits then $C C(\mathcal{F}) \in H_{m}\left(T_{A}^{*} Y, \mathbb{Z}\right)$. Recall that $C C$ is additive on distinguished triangles in $D(Y)$. This implies that the characteristic cycle map determines a homomorphism of abelian groups

$$
C C: K\left(D_{A}(Y)\right) \longrightarrow H_{m}\left(T_{A}^{*} Y, \mathbb{Z}\right) .
$$

We will denote by the same symbol the complexified homomorphism

$$
C C: K\left(D_{A}(Y)\right)_{\mathbb{C}} \longrightarrow H_{m}\left(T_{A}^{*} Y, \mathbb{C}\right) .
$$


Following [SV1] we will define intertwining functors on $D(X)$. If $w \in W$ write $l(w)$ for the length function. Let

$$
Y_{w} \subset X \times X
$$

be the variety of pairs of Borel subalgebras in the relative position $w$, and

$$
p_{1}, p_{2}: Y_{w} \longrightarrow X
$$

projections onto the first and second factors in $X \times X$. Then we define the intertwining functor attached to $w \in W$ by the formula:

$$
I_{w}=R p_{1 !} p_{2}^{-1}[l(w)]: D(X) \longrightarrow D(X) .
$$

One can show that $I_{w}$ is an equivalence of categories. Moreover, the equivalences $I_{w}$ induce an action of the Weyl group $W$ on the $K$-group $K(D(X))$. We write $[\mathcal{F}] \in K(D(X))$ for the image of an object $\mathcal{F}$ from $D(X)$. The action of $W$ on $K(D(X))$ will be denoted by

$$
w \cdot[\mathcal{F}]=\left[I_{w}(\mathcal{F})\right] .
$$

If $A \subset G$ the maps $p_{1}$ and $p_{2}$ are $A$-equivariant; hence the intertwining functors preserve $K\left(D_{A}(X)\right)$ and define a structure of $W$-module.

Proposition 3.1. The $K$-group $K\left(D_{P}(X)\right)$ is generated by standard sheaves $\mathcal{I}(D(w))=R i_{D(w) !}\left(\mathbb{C}_{D(w)}\right)$, where $w \in{ }^{\Theta} W$.

Proof. We know that $K\left(D_{P}(X)\right)$ is generated by standard sheaves $R i_{D(w) !}\left(\tau_{D(w)}\right)$, where $\tau_{D(w)}$ is an irreducible $P$-equivariant local system on the $P$-orbit $D(w)$ and $w \in W^{\Theta}$. Let $x \in D(w)$. The irreducible $P$-equivariant local systems on $D(w)$ are parametrized by the irreducible representations of the component group of $P \cap B_{x_{w}}$. On the other hand $P \cap B_{x_{w}}$ is connected [Bor2] so the only irreducible $P$-equivariant local system on $D(w)$ is the trivial sheaf.

Lemma 3.2. Let $w \in{ }^{\Theta} W$. Denote by e the unit element in $W$. Then the map $p_{2}$ induces a P-equivariant isomorphism of varieties

$$
p_{1}^{-1}(D(e)) \stackrel{\approx}{\longrightarrow} D(w) .
$$

Proof. The map $p_{1}$ is $G$-equivariant so we obtain

$$
p_{1}^{-1}\left(P . x_{0}\right)=P . p_{1}^{-1}\left(x_{0}\right)=P .\left(\left\{x_{0}\right\} \times B . x_{w}\right)=P .\left(B\left(x_{0} \times x_{w}\right)\right)=P .\left(x_{0} \times x_{w}\right) .
$$

Hence we have to show that $p_{2}$ induces a $P$-equivariant isomorphism of varieties

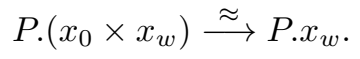

Let $w_{0} \in G$ be such that $A d\left(w_{0}\right)$ represents $w_{x_{0}}$. Denote by $\mathfrak{b}$ the Lie algebra of $B$ and by $\mathfrak{p}$ the Lie algebra of $P$. To prove the above statement it will suffice to show that

$$
\mathfrak{b} \cap A d\left(w_{0}\right) \mathfrak{b}=\mathfrak{p} \cap A d\left(w_{0}\right) \mathfrak{b} .
$$

To simplify notation denote by $\Delta_{0}$ the root system of the pair $(\mathfrak{g}, \mathfrak{c})$ and by $-\Delta_{0}^{+} \subset$ $\Delta_{0}$ the roots of $\mathfrak{b}$. Let $\Delta_{\Theta_{0}}$ be the root system spanned by the image $\Theta_{0}$ of $\Theta$ under the specialization $\tau_{x_{0}}^{*}$. By the choice of ${ }^{\Theta} W$ we have

$$
\left(-\Delta_{\Theta_{0}}^{+}\right) \cap w_{x_{0}}\left(-\Delta_{0}^{+}\right)=\emptyset .
$$


This implies further

$$
\begin{aligned}
\mathfrak{p} \cap A d\left(w_{0}\right) \mathfrak{b} & =\left\{\mathfrak{c}+\sum_{\alpha \in \Delta_{\Theta_{0}}} \mathfrak{g}_{\alpha}+\sum_{\alpha \in-\left(\Delta_{0}^{+}\right) \backslash \Delta_{\Theta_{0}}^{+}} \mathfrak{g}_{\alpha}\right\} \cap\left\{\mathfrak{c}+\sum_{\alpha \in w_{x_{0}}\left(-\Delta_{0}^{+}\right)} \mathfrak{g}_{\alpha}\right\} \\
& =\left\{\mathfrak{c}+\sum_{\alpha \in \Delta_{\Theta_{0}}^{+}} \mathfrak{g}_{\alpha}+\sum_{\alpha \in-\left(\Delta_{0}^{+}\right) \backslash \Delta_{\Theta_{0}}^{+}} \mathfrak{g}_{\alpha}\right\} \cap\left\{\mathfrak{c}+\sum_{\alpha \in w_{x_{0}}\left(-\Delta_{0}^{+}\right)} \mathfrak{g}_{\alpha}\right\} \\
& =\mathfrak{b} \cap A d\left(w_{0}\right) \mathfrak{b},
\end{aligned}
$$

as desired.

Proposition 3.3. Let $w \in{ }^{\Theta} W$. Denote by e the unit element in $W$. Then $I_{w}(\mathcal{I}(e))=\mathcal{I}(w)[l(w)]$. In particular, as a $W$-module $K\left(D_{P}(X)\right)$ is generated by $\mathcal{I}(e)$.

Proof. Let $i_{e}: D(e) \longrightarrow X$ and $j_{e}: p_{1}^{-1}(D(e)) \longrightarrow Y_{w}$ be the natural inclusions and let $p r_{1}: p_{1}^{-1}\left(D(e) \longrightarrow D(e)\right.$ and $p r_{2}: p_{1}^{-1}(D(e) \longrightarrow D(w)$ be projections. By the application of the base change to the Cartesian diagram

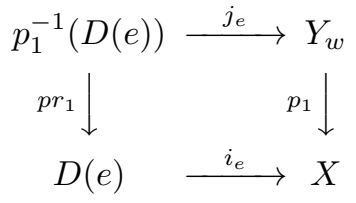

and the above lemma we obtain

$$
\begin{aligned}
I_{w}(\mathcal{I}(e)) & =R p_{2 !} p_{1}^{-1}\left(R i_{e !}\left(\mathbb{C}_{D(e)}\right)[l(w)]\right. \\
& =R p_{2 !} R j_{e !} p r_{1}^{-1}\left(\mathbb{C}_{D(e)}\right)[l(w)] \\
& =R p_{2 !} R j_{e !}\left(\mathbb{C}_{p_{1}^{-1} D(e)}\right)[l(w)] \\
& =R i_{w !} \mathbb{C}_{D(w)}[l(w)] \\
& =\mathcal{I}(w)[l(w)] .
\end{aligned}
$$

Consider now the $K$-group as a $W$-module for the action of intertwining operators, and consider the $W$-module structure on the homology group $H_{2 n}\left(T_{A}^{*} X, \mathbb{C}\right)$ defined by Rossmann. It was proved in [SV1] that the characteristic cycle map

$$
C C: K\left(D_{A}(X)\right)_{\mathbb{C}} \longrightarrow H_{2 n}\left(T_{A}^{*} X, \mathbb{C}\right)
$$

is a homomorphism of $W$-modules. By [Bo4, the characteristic cycles of standard sheaves form a basis (even over $\mathbb{Z}$ ) of $H_{2 n}\left(T_{A}^{*} X, \mathbb{C}\right)$. Thus we obtain the following result which will be the main ingredient in the proof of the limit formula.

Proposition 3.4. The cycle $C C\left(\mathcal{I}(D(e))\right.$ generates $H_{2 n}\left(T_{P}^{*} X, \mathbb{C}\right)$ as a $W$-module.

\section{Limit FORMULA}

Recall that $A=K$ or $A=G_{\mathbb{R}}$ and that $D_{A}(X)$ denotes the bounded equivariant derived category of $A$-equivariant sheaves on the flag variety $X$. There is an equivalence of categories

$$
\gamma: D_{K}(X) \longrightarrow D_{G_{\mathbb{R}}}(X)
$$


called the Matsuki correspondence for sheaves. The existence of this equivalence was conjectured by Kashiwara and proved in [MUV]. Schmid and Vilonen define the homomorphism [SV3, 3.7]

$$
\Phi: H_{2 n}\left(T_{K}^{*} X, \mathbb{C}\right) \longrightarrow H_{2 n}\left(T_{G_{\mathbb{R}}}^{*} X, \mathbb{C}\right),
$$

which makes the following diagram commutative

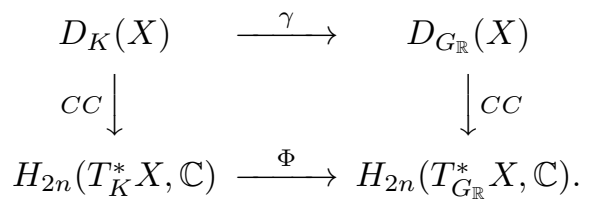

Put $\mathcal{N}_{k}=\cup\left\{G \cdot x: x \in \mathcal{N}, \operatorname{dim}_{\mathbb{C}} G \cdot x=2 k\right\}$ and $\overline{\mathcal{N}}_{k}=\cup_{l \leq k} \mathcal{N}_{l}$. The map $\Phi$ descends to the nilpotent cone by integrating cycles over the fibres of the moment map. At this point, we will use the identification $\mathfrak{g}^{*} \cong \mathfrak{g}$ defined by the Killing form. In particular, we view the moment map as the map

$$
\mu: T^{*} X \longrightarrow \mathfrak{g} \text {. }
$$

Let $\mathcal{V}^{\prime} \subset \mathcal{N}_{k} \cap \mathfrak{p}$ be a $K$-orbit, and $\mathcal{O}^{\prime} \subset \mathcal{N} \cap i \mathfrak{g}_{\mathbb{R}}$ a $G_{\mathbb{R}^{-o r b i t}}$. We orient $\mathcal{V}^{\prime}$ by the complex structure, and $\mathcal{O}^{\prime}$ by the Liouville form. We denote the corresponding cycles by $\left[\mathcal{V}^{\prime}\right]$ and $\left[\mathcal{O}^{\prime}\right]$. Here we use the assumption that $G_{\mathbb{R}}$ is connected. First we discuss the descent to $\mathcal{N} \cap \mathfrak{p}$. Recall that by the result of Kostant and Rallis $\mathrm{KR}$ the number of $K$-orbits in $\mathcal{N} \cap \mathfrak{p}$ is finite. Hence we may write

$$
\mathcal{N}_{k} \cap \mathfrak{p}=\mathcal{V}_{1} \cup \cdots \cup \mathcal{V}_{l},
$$

where $\mathcal{V}_{1}, \cdots, \mathcal{V}_{l}$ are $K$-orbits. The Mayer-Vietoris theorem for Borel-Moore homology implies

$$
H_{2 n}\left(\mu^{-1}\left(\mathcal{N}_{k} \cap \mathfrak{p}\right), \mathbb{C}\right)=\bigoplus_{j=1}^{l} H_{2 n}\left(\mu^{-1}\left(\mathcal{V}_{j}\right), \mathbb{C}\right)
$$

Given a cycle $C \in H_{2 n}\left(T_{K}^{*} X, \mathbb{Z}\right)$, let $k=k(C)$ be the minimal integer such that $C \in H_{2 n}\left(T_{K}^{*} X \cap \mu^{-1}\left(\overline{\mathcal{N}}_{k}\right), \mathbb{C}\right)$. Then $T_{K}^{*} X \cap \mu^{-1}\left(\mathcal{N}_{k}\right)$ is open in $T_{K}^{*} X \cap \mu^{-1}\left(\overline{\mathcal{N}}_{k}\right)$ and we consider the restriction

$$
C^{0}=\left.C\right|_{T_{K}^{*} X \cap \mu^{-1}\left(\mathcal{N}_{k}\right)} .
$$

By (12) we can write

$$
C^{0}=\sum_{j=1}^{l} C_{\mathcal{V}_{i}}, \quad C_{\mathcal{V}_{i}} \in H_{2 n}\left(\mu^{-1} \mathcal{V}_{i}, \mathbb{C}\right) .
$$

We draw now attention to (8) and denote by $p\left(\mathcal{C}, \mathcal{V}_{i}\right)$ the polynomial in $\mathcal{H}_{(n-k)}\left(\mathfrak{h}^{\star}\right)$ associated with the cycle $C_{\mathcal{V}_{i}}$.

We set

$$
\left(\operatorname{gr} \mu_{*}\right)_{K, \lambda}(C)=\sum_{i=1}^{l} p\left(C, \mathcal{V}_{i}\right)(\lambda)\left[\mathcal{V}_{i}\right] .
$$

Here we regard the left side as a top dimensional cycle in $\mathcal{N}_{k} \cap \mathfrak{p}$. In fact, by our assumption the orbits $\mathcal{V}_{i}$ are connected; hence the cycles $\left[\mathcal{V}_{i}\right], i=1, \cdots, l$ provide 
a basis of $H_{2 k}\left(\mathcal{N}_{k} \cap \mathfrak{p}, \mathbb{C}\right)$. In this way we obtain the map

$$
\left(\operatorname{gr} \mu_{*}\right)_{K, \lambda}: H_{2 n}\left(T_{K}^{*} X, \mathbb{C}\right) \longrightarrow \bigoplus_{k \geq 0} H_{2 k}\left(\mathcal{N}_{k} \cap \mathfrak{p}, \mathbb{C}\right)
$$

Next we consider the descent to $\mathcal{N} \cap i \mathfrak{g}_{\mathbb{R}}$. Set

$$
\mathcal{N}_{k} \cap i \mathfrak{g}_{\mathbb{R}}=\mathcal{O}_{1} \cup \cdots \cup \mathcal{O}_{l},
$$

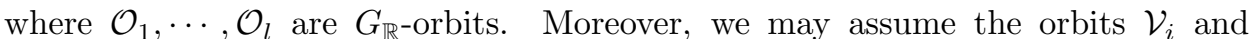
$\mathcal{O}_{i}$ are related by the Sekiguchi correspondence $\left[\mathrm{Se}\right.$. For $i=1, \ldots, l$ and $C^{\prime} \in$ $H_{2 n}\left(\mu^{-1}\left(\overline{\mathcal{N}}_{k}\right) \cap T_{G_{\mathbb{R}}}^{\star}, \mathbb{C}\right)$ we introduce polynomials $p\left(C^{\prime}, \mathcal{O}_{i}\right)$ analogously as $p\left(C, \mathcal{V}_{i}\right)$. The map

$$
\left(\operatorname{gr} \mu_{*}\right)_{G_{\mathbb{R}}, \lambda}: H_{2 n}\left(T_{G_{\mathbb{R}}}^{*} X, \mathbb{C}\right) \longrightarrow \bigoplus_{k \geq 0} H_{2 k}\left(\mathcal{N}_{k} \cap i \mathfrak{g}_{\mathbb{R}}, \mathbb{C}\right)
$$

is then defined by the formula

$$
\left(\operatorname{gr} \mu_{*}\right)_{G_{\mathbb{R}}, \lambda}\left(C^{\prime}\right)=\sum_{i=1}^{l} p\left(C^{\prime}, \mathcal{O}_{i}\right)(\lambda)\left[\mathcal{O}_{i}\right] .
$$

Schmid and Vilonen define a homomorphism [SV3, 5.10]

$$
\phi: \bigoplus_{k \geq 0} H_{2 k}\left(\mathcal{N}_{k} \cap \mathfrak{p}, \mathbb{C}\right) \longrightarrow \bigoplus_{k \geq 0} H_{2 k}\left(\mathcal{N}_{k} \cap i \mathfrak{g}_{\mathbb{R}}, \mathbb{C}\right),
$$

such that

$$
\left(\operatorname{gr} \mu_{*}\right)_{G_{\mathbb{R}}, \lambda} \circ \Phi=\phi \circ\left(\operatorname{gr} \mu_{*}\right)_{K, \lambda} .
$$

Moreover, they compute $\phi$ on the invariant part of the homology [SV3, 6.3]. In fact, suppose a $K$-orbit $\mathcal{V}^{\prime}$ and a $G_{\mathbb{R}}$-orbit $\mathcal{O}^{\prime}$ are related by the Sekiguchi correspondence. Then

$$
\phi\left(\left[\mathcal{V}^{\prime}\right]\right)=\left[\mathcal{O}^{\prime}\right] .
$$

Theorem 4.1 follows from the theory outlined above and from Ro3].

Theorem 4.1. Let $C \in H_{2 n}\left(T_{K}^{*} X, \mathbb{C}\right)$, and let $k=k(C)$ be the minimal integer such that $C \in H_{2 n}\left(T_{K}^{*} X \cap \mu^{-1}\left(\overline{\mathcal{N}}_{k}\right), \mathbb{C}\right)$. Then

$$
\Theta(\Phi(C), \lambda)=\sum_{i=1}^{l} p\left(C, \mathcal{V}_{i}\right)(\lambda) \Theta_{\mathcal{O}_{i}}+o\left(\lambda^{n-k}\right) .
$$

For $i=1, \ldots, l$ the map

$$
H_{2 n}\left(\mu^{-1}\left(\overline{\mathcal{N}}_{k}\right) \cap T_{K}^{\star} X, \mathbb{C}\right) \longrightarrow \mathcal{H}_{(n-k)}\left(\mathfrak{h}^{\star}\right), \quad C \mapsto p\left(C, \mathcal{V}_{i}\right)=p\left(\Phi(C), \mathcal{O}_{i}\right)
$$

is a homomorphism of $W$-modules.

Let $G_{\mathbb{R}}$ be a simple Lie group having a compact Cartan subgroup $T_{\mathbb{R}}$. Denote by $\mathfrak{t}_{\mathbb{R}}$ the Lie algebra of $T_{\mathbb{R}}$ and by $\mathfrak{t}$ the complexification of $\mathfrak{t}_{\mathbb{R}}$. Then there exists a positive root system

$$
\Delta^{+}(\mathfrak{g}, \mathfrak{t}) \subset \Delta(\mathfrak{g}, \mathfrak{c})
$$

such that exactly one simple root is noncompact and the multiplicity of this simple root in the highest root is one or two [Ch]. Denote by $\beta$ the unique simple noncompact root, and assume in addition that

$$
\text { the multiplicity of } \beta \text { in the highest root is one. }
$$


We remark that the choice of the positive root system with this property is related to the construction of the holomorphic discrete series [Ch. Denote by

$$
\mathfrak{b}_{x_{0}} \subset \mathfrak{g}
$$

the Borel subalgebra defined by $\left(\mathfrak{t},-\Delta^{+}(\mathfrak{g}, \mathfrak{t})\right.$ and by $B_{x_{0}}$ the corresponding Borel subgroup. Write

$$
\mathfrak{b}_{x_{0}}=\mathfrak{t}+\mathfrak{n}_{x_{0}}
$$

for the Levi decomposition. Let $\Theta_{x_{0}}=\Pi_{x_{0}} \backslash \beta$, and let

$$
\mathfrak{q} \supseteq \mathfrak{b}_{x_{0}}
$$

be the parabolic subalgebra defined by $-\Theta_{x_{0}}$ and

$$
Q \supseteq B_{x_{0}}
$$

the corresponding parabolic subgroup. Let

$$
\mathfrak{q}=\mathfrak{l}+\mathfrak{u} \text { and } Q=L U
$$

be the Levi decompositions. Any root $\gamma \in-\Delta^{+}(\mathfrak{g}, \mathfrak{t})$ of the form

$$
\gamma=\sum_{\alpha \in-\Theta_{x_{0}}} n_{\alpha} \alpha-\beta, n_{\alpha} \geq 0
$$

is necessarily noncompact. In fact, $\gamma$ can be written as

$$
\gamma=\alpha_{1}+\cdots+\alpha_{m},
$$

where $-\alpha_{i}$ is a simple root and $\alpha_{1}+\cdots+\alpha_{i}$ is also a root for any $1 \leq i \leq m$. We choose a root vector $X_{\alpha_{i}} \in \mathfrak{g}_{\alpha_{i}}$ and consider

$$
X_{\gamma}=\left[X_{\alpha_{m}},\left[X_{\alpha_{m-1}},\left[\cdots,\left[X_{\alpha_{2}}, X_{\alpha_{1}}\right] \cdots\right]\right]\right] .
$$

There exists a unique $i$ such that $\alpha_{i}=-\beta$; hence the expression for $X_{\gamma}$ implies that $X_{\gamma} \in \mathfrak{p}$, as desired.

The preceding discussion implies that

$$
\mathfrak{l}=\mathfrak{k}, \quad L=K \text { and } \mathfrak{u}=\mathfrak{n}_{x_{0}} \cap \mathfrak{p} .
$$

In particular, $\mathfrak{u}$ is $K$-invariant. Next we recall the result about the $K$-orbit decomposition of $\mathfrak{u}$ following $\mathrm{Ya}$. Denote by $r$ the real rank of $G_{\mathbb{R}}$. Then there exist $(r+1) K$-orbits $\mathcal{O}_{i}, 0 \leq i \leq r$, such that

$$
\mathfrak{p} \cap \mathfrak{n}_{x_{0}}=\mathcal{V}_{0} \cup \mathcal{V}_{1} \cup \cdots \cup \mathcal{V}_{r}, \quad \mathcal{V}_{0}=\{0\}, \quad \overline{\mathcal{V}}_{i}=\mathcal{V}_{0} \cup \cdots \cup \mathcal{V}_{i-1} \cup \mathcal{V}_{i}
$$

Here the closure is computed in Zariski (or in classical) topology. Denote by

$$
\mathcal{O}_{i} \subset \sqrt{-1} \mathfrak{g}_{\mathbb{R}}, \quad 0 \leq i \leq r,
$$

the $G_{\mathbb{R}^{-}}$orbit associated with the $K$-orbit $\mathcal{V}_{i}$ by the Sekiguchi correspondence. Our goal is to establish limit formulas for the orbits $\mathcal{O}_{i}$.

Define the orbits $Z=K \cdot x_{0}$ and $S=G_{\mathbb{R}} \cdot x_{0}$ and write

$$
i: Z \longrightarrow X \text { and } j: S \longrightarrow X
$$

for the inclusion maps. The orbit $Z$ is closed and the orbit $S$ is open in $X$ [Ma]. The pair $(Z, S)$ is a special case of the orbits related by the Matsuki correspondence.

Theorem 4.1 will be applied to the standard sheaves $R i_{*}\left(\mathbb{C}_{Z}\right)$ and $R j_{!}\left(\mathbb{C}_{S}\right)$. In this special case the Matsuki correspondence for sheaves is given by

$$
\gamma\left(R i_{*}\left(\mathbb{C}_{Z}\right)\right)=R j_{!}\left(\mathbb{C}_{S}\right)\left[2 \operatorname{codim}_{\mathbb{C}} Z\right] .
$$


We remark that the orientation of the conormal bundle $T_{Z}^{*} Y$ as a chain in [SV3, 4.34] differs precisely by the factor $(-1)^{p}$, where

$$
p=\operatorname{dim}_{\mathbb{C}} \mathfrak{n}_{x_{0}} \cap \mathfrak{p},
$$

from the orientation induced by the complex structure. By (11) this implies further

$$
\Phi\left((-1)^{p}\left[T_{Z}^{*} Y\right]\right)=C C\left(R j_{!}\left(\mathbb{C}_{S}\right)\right) .
$$

Proposition 4.2. The cycle $\left[T_{Z}^{*} X\right]$ generates $H_{2 n}\left(\mu^{-1}(\mathfrak{u}), \mathbb{C}\right)$ as a $W$-module.

Proof. We have

$$
Q \cdot x_{0}=K \cdot x_{0}=Z \text { and } T_{Q}^{*} X=\mu^{-1}\left(\mathfrak{q}^{\perp}\right)=\mu^{-1}(\mathfrak{u}) .
$$

The claim of the proposition follows now from Proposition 3.4 .

Let $\mathcal{W}$ be a nilpotent $G$-orbit and $\nu \in \mathcal{W}$. Given a $W$-module $V$ consider the projection $P_{\chi_{\nu}}$ to the isotypical component of type $\chi_{\nu}$ :

$$
P_{\chi_{\nu}}: V \longrightarrow V, \quad P_{\chi_{\nu}}(\Gamma)=\frac{\operatorname{deg} \chi_{\nu}}{|W|} \sum_{w \in W} \chi_{\nu}\left(w^{-1}\right) w \Gamma .
$$

Lemma 4.3. Let $\nu_{i} \in \mathcal{V}_{i}$ or $\nu_{i} \in \mathcal{O}_{i}, 0 \leq i \leq r$. Then

$$
P_{\chi_{\nu_{i}}}\left(H_{2 n}\left(\mu^{-1}(\overline{\mathcal{V}})\right)\right) \subset H_{2 n}\left(\mu^{-1}\left(\overline{\mathcal{V}}_{i}\right)\right) \text { or } P_{\chi_{\nu_{i}}}\left(H_{2 n}\left(\mu^{-1}(\overline{\mathcal{O}})\right)\right) \subset H_{2 n}\left(\mu^{-1}\left(\overline{\mathcal{O}}_{i}\right)\right) \text {. }
$$

Proof. We prove only the formula for the $K$-orbits, the case of $G_{\mathbb{R} \text {-orbits being }}$ analogous. For $i<m \leq r$ consider the exact sequence of $W$-modules

$$
0 \longrightarrow H_{2 n}\left(\mu^{-1}\left(\overline{\mathcal{V}}_{m-1}\right)\right) \longrightarrow H_{2 n}\left(\mu^{-1}\left(\overline{\mathcal{V}}_{m}\right)\right) \longrightarrow H_{2 n}\left(\mu^{-1}\left(\mathcal{V}_{m}\right)\right) \longrightarrow 0
$$

Recall that

$$
H_{2 n}\left(\mu^{-1}\left(\mathcal{V}_{m}\right), \mathbb{C}\right) \cong H_{2 d}\left(\mu^{-1}(\nu), \mathbb{C}\right)^{C_{K}\left(\nu_{m}\right)} .
$$

Using the Springer parametrization of Weyl group representations we deduce that $P_{\chi_{\nu_{i}}}(C) \mid \mu^{-1}\left(\mathcal{V}_{m}\right)=0$ for $m>i$ and $C \in H_{2 n}\left(\mu^{-1}\left(\overline{\mathcal{V}}_{m}\right)\right)$. This implies

$$
P_{\chi_{\nu_{i}}} H_{2 n}\left(\mu^{-1}\left(\overline{\mathcal{V}}_{m}\right)\right) \subset H_{2 n}\left(\mu^{-1}\left(\overline{\mathcal{V}}_{m-1}\right)\right),
$$

and furthermore because of $P_{\chi_{\nu_{i}}}^{2}=P_{\chi_{\nu_{i}}}$

$$
P_{\chi_{\nu_{i}}} H_{2 n}\left(\mu^{-1}\left(\overline{\mathcal{V}}_{m}\right)\right) \subset P_{\chi_{\nu_{i}}} H_{2 n}\left(\mu^{-1}\left(\overline{\mathcal{V}}_{m-1}\right)\right)
$$

Combining these inclusions we obtain the conclusion of the lemma.

Theorem 4.4 is a variant of Rossmann's character formula proved by Schmid and Vilonen [SV2, Bo2. It will play an important role in the study of asymptotic properties of elliptic orbital integrals. Recall that $\lambda_{x_{0}} \in i \mathfrak{t}_{\mathbb{R}}^{*}$ is dominant if

$$
\left(\lambda_{x_{0}}, \alpha\right)>0 \text { for any } \alpha \in \Delta\left(\mathfrak{g} / \mathfrak{b}_{x_{0}}, \mathfrak{t}\right) .
$$

Theorem 4.4. Let $G_{\mathbb{R}}$ be a connected, linear, semisimple Lie group. Consider the Borel subalgebra $\mathfrak{b}_{x_{0}}$ defined in (20) and the open orbit $S=\operatorname{Ad}\left(G_{\mathbb{R}}\right) \cdot x_{0}$. Let $\phi \in C_{c}^{\infty}\left(\mathfrak{g}_{\mathbb{R}}\right)$. Denote by $\hat{\phi}$ the Fourier transform of $\phi$. Then for $\lambda \in \mathfrak{h}^{* \prime}$ such that $\lambda_{x_{0}}$ is dominant we have

$$
\int_{C C\left(R j_{!} \mathbb{C}_{S}\right)} \mu_{\lambda}^{*}\left(\hat{\phi} \sigma_{\lambda}^{m}\right)=(-1)^{p} \int_{G_{\mathbb{R}} \cdot \lambda_{x_{0}}} \hat{\phi} \sigma_{\lambda}^{m} .
$$


Let $\pi$ be an irreducible representation of $G_{\mathbb{R}}$. We will denote by $\Theta_{\pi}$ the character of $\pi$ and by $\theta_{\pi}$ the pullback of $\Theta_{\pi}$ on $\mathfrak{g}_{\mathbb{R}}$ under the exponential map.

Let $\lambda_{x_{0}} \in i \mathfrak{t}_{\mathbb{R}}^{*}$ be regular and dominant. Assume in addition that $\lambda_{x_{0}}+\rho_{x_{0}}$ lifts to a character of $T_{\mathbb{R}}$. Then $\lambda$ determines a discrete series representation $\pi_{\lambda}$. We relate now the orbit $\mathcal{O}$ and the wave front set $W F\left(\pi_{\lambda}\right)$.

Proposition 4.5. If $\pi_{\lambda}$ is a discrete series representation defined by $\lambda_{x_{0}} \in i \mathrm{t}_{\mathbb{R}}^{*}$ then we have

$$
W F\left(\pi_{\lambda}\right)=\overline{\mathcal{O}} .
$$

Proof. Observe that $C C\left(R i_{\star} \mathbb{C}_{Z}\right)$ is supported on the conormal bundle $T_{Z}^{*} X$ and that $\mu\left(T_{Z}^{*} X\right)=\overline{\mathcal{V}}$ for a unique $K$-orbit $\mathcal{V} \subset \mathcal{N} \cap \mathfrak{p}$. Using these facts we deduce

$$
\left(g r \mu_{\star}\right)_{K, \lambda}\left(C C\left(R i_{\star} \mathbb{C}_{Z}\right)\right)=p_{\mathcal{V}}(\lambda)[\mathcal{V}] .
$$

By the assumption $\lambda$ is dominant; hence it follows from [SV3, 7.5] that $p_{\mathcal{V}}(\lambda) \neq 0$. For $t \in \mathbb{R}_{>0}$ denote by $m_{t}: \mathfrak{g} \longrightarrow \mathfrak{g}$ the map $m_{t}(\xi)=t \xi, \xi \in \mathfrak{g}$. Recall that

$$
d m_{\mathcal{O}}=\left(\sigma_{\mathcal{O}}\right)^{2 k} /(2 \pi i)^{k} k !, 2 k=\operatorname{dim}_{\mathbb{R}} \mathcal{O}
$$

is the canonical measure on $\mathcal{O}$. By the main result of SV3 the leading term of $m_{t}^{*} \theta_{\pi_{\lambda}}$ at $t \rightarrow 0$ is the Fourier transform of $p_{\mathcal{O}}(\lambda) \cdot d m_{\mathcal{O}}$, where

$$
\left(g r \mu_{\star}\right)_{G_{\mathbb{R}}, \lambda}\left(C C\left(R j_{!} \mathbb{C}_{S}\right)\right)=p_{\mathcal{O}}(\lambda)[\mathcal{O}],
$$

and $p_{\mathcal{O}}(\lambda)=p_{\mathcal{V}}(\lambda) \neq 0$. On the other hand Rossmann proves [Ro3] that the wave front of an irreducible representation $\pi$ is the union of closures of the leading orbits in $m_{t}^{*} \theta_{\pi_{\lambda}}$ as $t \rightarrow 0$. In particular, $W F\left(\pi_{\lambda}\right)=\overline{\mathcal{O}}$.

Now we are ready to prove the main result of the paper.

Theorem 4.6. Let $G_{\mathbb{R}}$ be a connected linear semisimple Lie group and let $\mathfrak{t}_{\mathbb{R}}$ be a compact Cartan subalgebra. Suppose that $G_{\mathbb{R}}$ has holomorphic discrete series $\pi_{\mu}$, where $\mu \in i \mathfrak{t}_{\mathbb{R}}$ is regular. Let $C \subset i \mathfrak{k}_{\mathbb{R}}$ be the chamber for which $\mu$ is dominant. Let $\mathcal{O} \subset i \mathfrak{g}_{\mathbb{R}}^{*}$ be a nilpotent coadjoint $G_{\mathbb{R}}$-orbit such that $W F\left(\pi_{\mu}\right)=\overline{\mathcal{O}}$. Then for any nilpotent $G_{\mathbb{R}}$-orbit $\mathcal{O}_{i} \subset \overline{\mathcal{O}}$ there exists up to a constant unique harmonic polynomial $p \in \mathbb{C}[\mathfrak{h}]$ corresponding to the $W$-character $\chi_{\nu}, \nu \in \mathcal{O}_{i}$, such that the following limit formula for the orbital measures holds

$$
\lim _{\lambda \rightarrow 0(C)} p(\partial) m_{\lambda}=\kappa m_{\mathcal{O}_{i}} .
$$

Here $\kappa$ is a nonzero constant.

Proof. Observe first that $\Phi\left(H_{2 n}\left(\mu^{-1}(\overline{\mathcal{V}}), \mathbb{C}\right)\right) \subset H_{2 n}\left(T_{G_{\mathbb{R}}}^{\star} X, \mathbb{C}\right)$. Set

$$
\Gamma_{1}=C C\left(R i_{\star} \mathbb{C}_{Z}\right), \quad \Gamma_{2}=C C\left(R j_{!} \mathbb{C}_{S}\right) .
$$

Then $\Phi\left(\Gamma_{1}\right)=\Gamma_{2}$. By the result of Rossmann [Ro3, 2.4.2], $\mu\left(\operatorname{supp}\left(\Gamma_{2}\right)\right)$ is equal to the wave front set of the distribution $\Theta\left(\Gamma_{2}, \lambda\right)$. On the other hand, (14) and (15) imply that $\mu\left(\operatorname{supp}\left(\Gamma_{2}\right)\right)=\overline{\mathcal{O}}$. In particular, we have $\Gamma_{2} \in H_{2 n}\left(\mu^{-1}(\overline{\mathcal{O}}), \mathbb{C}\right)$. Recall that $\Gamma_{1}$ generates $H_{2 n}\left(\mu^{-1}(\overline{\mathcal{V}}), \mathbb{C}\right)$ as a $W$-module; hence we have $\Phi\left(H_{2 n}\left(\mu^{-1}(\overline{\mathcal{V}}), \mathbb{C}\right)\right)$ $\subset H_{2 n}\left(\mu^{-1}(\overline{\mathcal{O}}), \mathbb{C}\right)$. The equality of dimensions of $H_{2 n}\left(\mu^{-1}(\overline{\mathcal{V}}), \mathbb{C}\right)$ and $H_{2 n}\left(\mu^{-1}(\overline{\mathcal{O}})\right.$, $\mathbb{C})$ gives finally

$$
\Phi\left(H_{2 n}\left(\mu^{-1}(\overline{\mathcal{V}}), \mathbb{C}\right)\right)=H_{2 n}\left(\mu^{-1}(\overline{\mathcal{O}}), \mathbb{C}\right)
$$


Let $\nu \in \mathcal{O}_{i}$. By (12) $\Gamma_{2}$ generates $H_{2 n}\left(\mu^{-1}(\overline{\mathcal{O}}), \mathbb{C}\right)$ as a $W$-module; hence $P_{\chi_{\nu}} \Gamma_{2} \neq 0$. Then $r=b\left(P_{\chi_{\nu}} \Gamma_{2}\right) \neq 0$ and applying (13) we obtain

$$
\Theta\left(P_{\chi_{\nu}} \Gamma_{2}, \lambda\right)=r(\lambda) \Theta_{\mathcal{O}_{i}}+o\left(\lambda^{d}\right), d=\operatorname{dim}_{\mathbb{C}} \mu^{-1}(\nu) .
$$

Set $p=\overline{\delta(r)}$. Then by (2) $p$ is a harmonic polynomial on $\mathfrak{h}$ corresponding to the $W$-character $\chi_{\nu}$. Moreover, the definition of $p$ implies

$$
p(\partial) r(\lambda)=p(\partial) r(0) \neq 0 .
$$

Now we apply (3) to conclude

$$
\lim _{\lambda \rightarrow 0(C)} p(\partial) \Theta\left(\Gamma_{2}, \lambda\right)=p(\partial) r(0) \Theta_{\mathcal{O}} .
$$

Here we used the formula $\chi_{\nu}\left(w^{-1}\right)=\chi_{\nu}(w), w \in W$, which is a consequence of the fact that $\chi_{\nu}$ is defined over $\mathbb{Q}[\mathrm{BM}$, Th. 3]. To complete the proof it will suffice to use (14), and take the inverse Fourier transform.

\section{REFERENCES}

[BL] Joseph Bernstein and Valery Lunts, Equivariant sheaves and functors, Lecture Notes in Mathematics, vol. 1578, Springer-Verlag, Berlin, 1994, DOI 10.1007/BFb0073549. MR 1299527

[Bor1] Armand Borel, Sur la cohomologie des espaces fibrés principaux et des espaces homogènes de groupes de Lie compacts (French), Ann. of Math. (2) 57 (1953), 115-207, DOI 10.2307/1969728. MR51508

[Bor2] Armand Borel, Linear algebraic groups, 2nd ed., Graduate Texts in Mathematics, vol. 126, Springer-Verlag, New York, 1991, DOI 10.1007/978-1-4612-0941-6. MR.1102012

[BM] Walter Borho and Robert MacPherson, Représentations des groupes de Weyl et homologie d'intersection pour les variétés nilpotentes (French, with English summary), C. R. Acad. Sci. Paris Sér. I Math. 292 (1981), no. 15, 707-710. MR618892

[Bo1] Mladen Božičević, A limit formula for elliptic orbital integrals, Duke Math. J. 113 (2002), no. 2, 331-353, DOI 10.1215/S0012-7094-02-11325-8. MR1909221

[Bo2] Mladen Božičević, Characteristic cycles of standard sheaves associated with open orbits, Proc. Amer. Math. Soc. 136 (2008), no. 1, 367-371, DOI 10.1090/S0002-9939-07-08986-1. MR 2350425

[Bo3] Mladen Božičević, Limit formulas for groups with one conjugacy class of Cartan subgroups (English, with English and French summaries), Ann. Inst. Fourier (Grenoble) 58 (2008), no. 4, 1213-1232. MR2427959

[Bo4] Mladen Božičević, Homology groups of conormal varieties, Mediterr. J. Math. 4 (2007), no. 4, 407-418, DOI 10.1007/s00009-007-0126-x. MR2369235

[Ch] Jen-Tseh Chang, Characteristic cycles of holomorphic discrete series, Trans. Amer. Math. Soc. 334 (1992), no. 1, 213-227, DOI 10.2307/2153979. MR.1087052

[KR] B. Kostant and S. Rallis, Orbits and representations associated with symmetric spaces, Amer. J. Math. 93 (1971), 753-809, DOI 10.2307/2373470. MR311837

[KS] Masaki Kashiwara and Pierre Schapira, Sheaves on manifolds, Grundlehren der Mathematischen Wissenschaften [Fundamental Principles of Mathematical Sciences], vol. 292, Springer-Verlag, Berlin, 1990. With a chapter in French by Christian Houzel, DOI 10.1007/978-3-662-02661-8. MR.1074006

[Ma] Toshihiko Matsuki, The orbits of affine symmetric spaces under the action of minimal parabolic subgroups, J. Math. Soc. Japan 31 (1979), no. 2, 331-357, DOI 10.2969/jmsj/03120331. MR527548

[Mi] D. Miličić, Localization and representation theory of reductive Lie groups, Preliminary manuscript, 1993.

[MUV] I. Mirković, T. Uzawa, and K. Vilonen, Matsuki correspondence for sheaves, Invent. Math. 109 (1992), no. 2, 231-245, DOI 10.1007/BF01232026. MR1172690 
[Ro1] W. Rossmann, Nilpotent orbital integrals in a real semisimple Lie algebra and representations of Weyl groups, Operator algebras, unitary representations, enveloping algebras, and invariant theory (Paris, 1989), Progr. Math., vol. 92, Birkhäuser Boston, Boston, MA, 1990, pp. 263-287. MR.1103593

[Ro2] W. Rossmann, Picard-Lefschetz theory for the coadjoint quotient of a semisimple Lie algebra, Invent. Math. 121 (1995), no. 3, 531-578, DOI 10.1007/BF01884311. MR.1353308

[Ro3] W. Rossmann, Picard-Lefschetz theory and characters of a semisimple Lie group, Invent. Math. 121 (1995), no. 3, 579-611, DOI 10.1007/BF01884312. MR.1353309

[SV1] Wilfried Schmid and Kari Vilonen, Characteristic cycles of constructible sheaves, Invent. Math. 124 (1996), no. 1-3, 451-502, DOI 10.1007/s002220050060. MR.1369425

[SV2] Wilfried Schmid and Kari Vilonen, Two geometric character formulas for reductive Lie groups, J. Amer. Math. Soc. 11 (1998), no. 4, 799-867, DOI 10.1090/S0894-0347-98-002756. MR 1612634

[SV3] Wilfried Schmid and Kari Vilonen, Characteristic cycles and wave front cycles of representations of reductive Lie groups, Ann. of Math. (2) 151 (2000), no. 3, 1071-1118, DOI 10.2307/121129. MR1779564

[Se] Jirō Sekiguchi, Remarks on real nilpotent orbits of a symmetric pair, J. Math. Soc. Japan 39 (1987), no. 1, 127-138, DOI 10.2969/jmsj/03910127. MR867991

[Ya] Hiroshi Yamashita, Cayley transform and generalized Whittaker models for irreducible highest weight modules (English, with English and French summaries), Astérisque 273 (2001), 81-137. Nilpotent orbits, associated cycles and Whittaker models for highest weight representations. MR 1845715

Department of Geotechnical Engineering, University of Zagreb, Hallerova 7, 42000 VARAŽdin, CROATIA

Email address: mladen.bozicevic@gmail.com 